\title{
Direct Numerical Simulations of Turbulent Channel Flows with Moving Wall
}

\author{
X. $\operatorname{Wan}^{1}$, S. W. $\mathrm{Ma}^{2}$, J. B. Zhang ${ }^{1 *}$, C. H. $\mathrm{Li}^{1}$
}

${ }^{1}$ School of Aeronautic Science and Technology, Beijing University of Aeronautics and Astronautics, Beijing 100083, China

${ }^{2}$ School of Astronautics, Beijing University of Aeronautics and Astronautics, Beijing 100083, China

Email: Zhangjb@buaa.edu.cn

\begin{abstract}
Direct numerical simulations of turbulent channel flows with moving spanwise wall is performed in this paper. The aim of this work is to show what influences can be caused in turbulent channel flow by spanwise moving wall, that introduces a new characteristic velocity. Figure 1 shows the flow geometry and the coordinate system. The DNS was performed using the standard spectral method. Three cases with the spanwise wall velocity $W=0, U_{m}$ and $2 U_{m}$ are simulated at constant flow rate, here $U_{m}$ is the mean stream velocity. The necessary parameters for DNS are as follows: $R e_{m}=U_{m} \delta / \nu=2800$ $\left(R e_{\tau 0}=u_{\tau 0} \delta / \nu \approx 180\right)$, the computational domain is $4 \pi \delta, 2 \delta$ and $2 \pi \delta$ for the streamwise $(x)$, normal $(y)$ and spanwise $(z)$ directions, respectively, and the grid resolution is $128 \times 129 \times 128$. The results show that spanwise moving wall induce the skin friction increasing. The second-order turbulence statistics such as root-mean-square velocity fluctuations, Reynolds shear stress and root-mean-square vorticity fluctuations are substantial increase with spanwise velocity increasing. It is also observed that the root-mean-square velocity fluctuations become more even across the channel with spanwise velocity increasing.
\end{abstract}

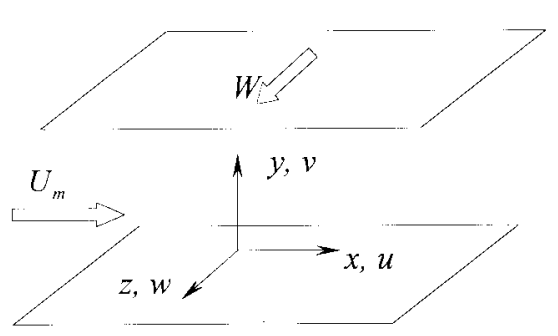

Figure 1: Flow geometry and coordinate system

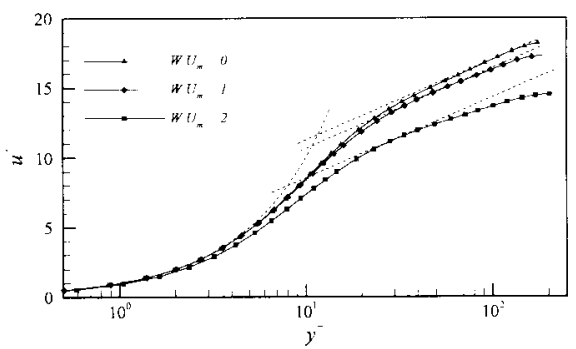

Figure 2: Mean streamwise velocity profiles

\section{REFERENCES}

1. Xu CX, Huang WX. Transient response of Reynolds stress transport to spanwise wall oscillation in a turbulent channel flow. Physics of Fluids, 2005;17:(1) Art. No. 018101

2. Min T, Yoo JY, Choi H, Joseph DD. Drag reduction by polymer additives in a turbulent channel flow. J. Fluid Mech., 2003;486:213-238

3. Karniadakis GE, Israeli M, Orszag SA. High-order splitting methods for the incompressible NavierStokes equations. J. Comput. Phys., 1991;97(2):414-443 